\title{
Degradación de la respuesta en frecuencia de fotodiodos en mediciones de señales ópticas intensas
}

\author{
Gastón Arrigo*, Patricio Sorichetti* y Eduardo Acosta*1 \\ *Universidad de Buenos Aires, Facultad de Ingeniería, \\ Grupo de Láser, Óptica de Materiales y Aplicaciones Electromagnéticas (GLOMAE) \\ Paseo Colón 850, C1063ACV, Buenos Aires, Argentina \\ ${ }^{1}$ eacosta@fi.uba.ar \\ Recibido: 10/07/17; Aceptado: 11/11/17
}

\begin{abstract}
In this paper, we present measurements that reveal changes in the frequency response of a PIN-type photodetector with the increase of the intensity of the light reaching the detector. In particular, two effects are observed at high illumination levels: the bandwidth of the detector decreases and the phase response is modified. For low intensities the bandwidth follows a power law with exponent -1 with the average current of the photodetector, but at high illumination the exponent tends to $\mathbf{- 0 . 5}$.
\end{abstract}

Resumen- En este trabajo se presentan mediciones que revelan cambios en la respuesta en frecuencia de un fotodetector tipo PIN debida al cambio en la intensidad de la luz que llega al detector. En particular, se observan dos efectos a altos niveles de iluminación: el ancho de banda del detector disminuye y se modifica su respuesta de fase. Para bajas intensidades el ancho de banda sigue una ley de potencias con exponente $\mathbf{- 1}$ con la corriente media del fotodetector, pero en alta iluminación el exponente tiende a -0,5.

\section{INTRODUCCIÓN}

Actualmente existen numerosas publicaciones que describen la construcción y características de funcionamiento de fotodetectores en el régimen de detección de pequeñas señales ópticas, así como también sobre el diseño de detectores para señales ópticas con modulación digital o para modulación de intensidad de alto índice (mayor al 30\%) [1], [2]. Sin embargo, no abunda la bibliografía que facilite la implementación de fotodetectores en aplicaciones que involucren rápidos pero pequeños cambios de amplitud de una señal óptica de elevada potencia media [3]. Tal es el caso de la detección de señales ópticas moduladas en amplitud, con baja profundidad de modulación y gran ancho de banda, como cuando se estudian fluctuaciones en láseres de potencia, control de distorsión en enlaces multicanales analógicos de fibra óptica (bajo condiciones de carga variable) [4] y sistemas electroópticos de medición [5], [6].

Los dispositivos cuánticos con ganancia intrínseca como los fotomultiplicadores y los fotodiodos de avalancha se utilizan generalmente en aplicaciones de conteo de fotones o bien cuando se desea detectar una señal óptica débil, ya que su diseño está optimizado para trabajar con niveles bajos de radiación incidente.

Los fabricantes suelen especificar la máxima intensidad óptica de entrada $S_{M A X}\left(\mathrm{en} \mathrm{W} / \mathrm{m}^{2}\right.$ ) para que el dispositivo funcione linealmente, el tiempo de trepada (rise time) y de caída (fall time) medidos con pulsos ópticos de bajo nivel (cuya intensidad es bastante inferior al límite de linealidad). Sin embargo la respuesta en frecuencia para los distintos valores de intensidad óptica no es informada.

Si la señal óptica es inferior a $S_{M A X}$, los portadores generados en la juntura son transportados por el campo eléctrico en la zona intrínseca con un tiempo de tránsito característico independiente de la intensidad óptica $S$ y la corriente entregada $I$ es proporcional a esta. Al aumentar el nivel de intensidad óptica $\left(S>S_{M A X}\right)$, las densidad de portadores libres aumenta y, si $S$ es lo suficientemente grande el campo eléctrico en la juntura del fotodiodo PIN deja de ser uniforme; en esta condición, los portadores de carga se acumulan en las zonas no desiertas generando una caída de potencial opuesta a la tensión de polarización y provocando una disminución del campo eléctrico con el consecuente incremento en los tiempos de arrastre de portadores. Esto tiene como resultado una degradación de la respuesta en frecuencia y por lo tanto una disminución en el ancho de banda del dispositivo [7]. Se ha demostrado, mediante mediciones experimentales y simulaciones, que niveles de intensidad aún mayores no sólo afectan el tiempo de respuesta sino también la linealidad del diodo. Para evitar estos efectos se ha propuesto construir otras arquitecturas, tales como fotodetectores distribuidos en una guía de onda (traveling-wave photodetector, TWPD) y los fotodiodos iluminados en superficie (surface illuminated photodiode, SIPD) [8] [9].

Los diferentes tipos de fotodetectores de alta frecuencia, aptos para señales de alta potencia, presentan variaciones de la frecuencia de corte en función de la corriente media. La relación entre la corriente media $I_{\text {med }}$ (normalizada respecto a la corriente de saturación $I_{\text {sat }}$ ) con la frecuencia de corte $f_{3 d B}$ sigue la forma:

$$
f_{3 b B} \sim\left(I_{\text {med }} / I_{\text {sat }}\right)^{-\alpha}
$$

donde el valor de $\alpha$ depende del tipo de fotodetector, $\alpha=1$ corresponde a fotodetectores TWPD ó $\alpha=0,5$ para fotodiodos SIPD [10].

En muchos trabajos de procesamiento de señales suponen que el detector es ideal y en particular no se tiene en cuenta la variación del ancho de banda del detector con el nivel medio de la señal de entrada.

En este trabajo se presentan los resultados obtenidos en la 
caracterización de un fotodiodo PIN comercial en función de la intensidad de luz media $S_{m e d}$ y para un rango de frecuencias de modulación de la portadora óptica entre $1 \mathrm{kHz}$ y $10 \mathrm{MHz}$.

Se describe primero la caracterización de la fuente luminosa con modulación armónica y luego los experimentos de caracterización del fotodiodo. Al final se discute las dificultades en la recuperación de señales con forma arbitraria que introduce la alinealidad por cambios en la intensidad media de la luz.

\section{PARTE EXPERIMENTAL}

\section{A. Dispositivo emisor}

Como fuente de referencia, se utilizó un módulo láser de $3 \mathrm{~mW}(670 \mathrm{~nm})$ marca Global Laser modelo 1060-42-000 (RS 194-004). Este dispositivo permite modular la intensidad lumínica empleando señales analógicas con frecuencias de $100 \mathrm{~Hz}$ hasta $50 \mathrm{MHz}$. El mismo fue alimentado con una fuente ad-hoc diseñada y construida en el laboratorio.

Se utilizó un generador de señal analógico GW Instek SFG-830 conectado a la entrada de $50 \Omega$ del módulo láser para producir señales ópticas con una profundidad de modulación del $2 \%$.

Para la caracterización de la respuesta en frecuencia del módulo láser se montó el sistema de la Figura 1. La señal óptica producida por el láser atraviesa un arreglo óptico de lente y atenuador antes de llegar al detector.

El detector empleado (Thorlabs modelo PDA155) consta de un fotodetector de silicio de alta velocidad seguido de una etapa amplificadora, con ancho de banda de $50 \mathrm{MHz}$ y una salida máxima de 5V. Este dispositivo se presenta como un módulo cerrado con alimentación externa y una salida tipo BNC para el monitoreo de la señal. De acuerdo a su hoja de datos, si utilizáramos la luz del láser sin atenuar $(3 \mathrm{~mW})$ obtendríamos a la salida del mismo una tensión superior a la admitida por el dispositivo; esto nos advierte que estaríamos trabajando con un nivel superior al soportado que podría dañarlo de forma permanente. Hay que considerar que el ancho de banda especificado por el fabricante es de $50 \mathrm{MHz}$ y corresponde a una señal de luz que produce una salida media de $200 \mathrm{mV}$ con una modulación del $100 \%$.

Los atenuadores empleados fueron construidos a partir de placas de acrílico de distinto espesor y color. Se determinó experimentalmente que las atenuaciones del conjunto de filtros empleados fueron $3,6 \mathrm{~dB} ; 7,3 \mathrm{~dB} ; 14,5 \mathrm{~dB} ; 25,5 \mathrm{~dB}$; $29 \mathrm{~dB}$ y $38 \mathrm{~dB}$.

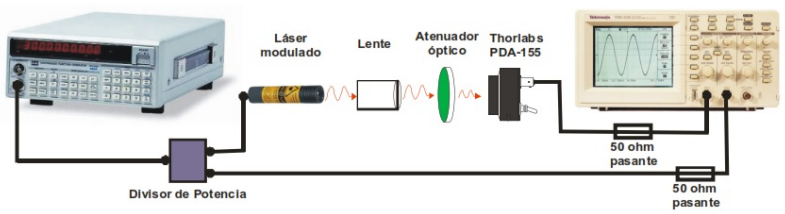

Fig. 1. Disposición de elementos para la caracterización del módulo láser

Se empleó un osciloscopio Tektronik TDS210 para determinar la transferencia del sistema, definida como la relación entre las tensiones de pico del PDA155 y la señal del generador. Los resultados se presentan en la Figura 2.
A fin de caracterizar el comportamiento del módulo láser se usó el filtro de $38 \mathrm{~dB}$ de modo que la señal pueda medir con comodidad pero sin salir del rango de señales pequeñas especificado por el fabricante $\left(S<S_{M A X}\right)$.

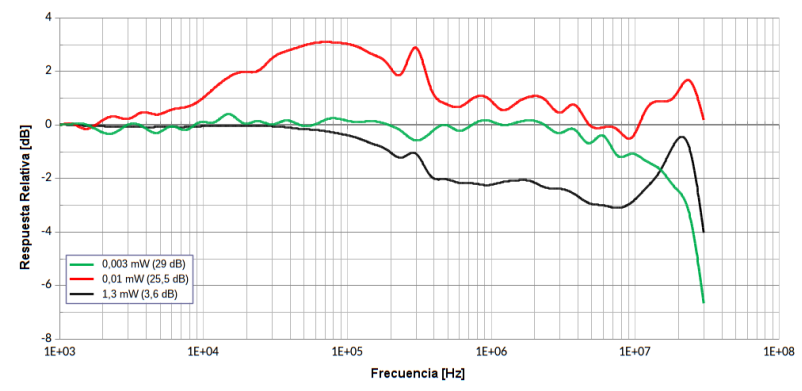

Fig. 2. Módulo de la transferencia para el PDA155 para diferentes intensidades medias.

En la Figura 2 se representa la respuesta relativa de amplitud (referida a $1 \mathrm{KHz}$ ) en $\mathrm{dB}$. Para la señal de menor intensidad (con el filtro de 29dB) el PDA155 se comporta según las especificaciones del fabricante. Al aumentar la intensidad se observa una dependencia mas compleja con la frecuencia con apartamientos que llegan hasta $\pm 2 \mathrm{~dB}$ restringiendo el uso de este dispositivo en aplicaciones de metrología.

\section{B. Caracterización del fotodiodo}

El fotodiodo caracterizado fue el BPW24R (Vishay), con encapsulado TO-18 cubierto con una lente de vidrio, con un rango espectral de $600 \mathrm{~nm}$ a $1050 \mathrm{~nm}, 50 \mathrm{MHz}$ de ancho de banda $\left(f_{B W}=1 /\left(2 \pi R_{L} C_{j}\right) \simeq 0,35 / t_{\text {rise }}\right), 12^{\circ}$ de apertura, área sensible de $0,78 \mathrm{~mm}^{2}$ y $3,2 \mathrm{pF}$ de capacidad en condiciones de polarización inversa con $10 \mathrm{~V}$.

En la Figura 3 se muestra el circuito empleado para caracterizar la respuesta en frecuencia del fotodiodo. El filtro pasa bajos de tres etapas se incluye para atenuar las altas frecuencias que pudieran ser inducidas por la fuente de alimentación. El terminal del cátodo se conectó directamente a la salida BNC con el fin de medir la señal de salida sin ningún tipo de amplificación.

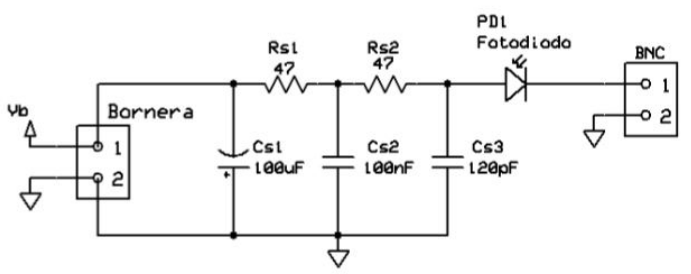

Fig. 3. Circuito con filtro y fotodiodo para la medición de la respuesta en frecuencia

Para determinar la corriente entregada por el fotodiodo $I$ al cambiar la tensión inversa de polarización $V$ y para distintas $S_{m e d}$, se usó el arreglo experimental de la Figura 4. En este experimento se trabajó con el módulo láser sin modular. La lente se empleó para focalizar el haz del láser sobre el fotodiodo. Una impedancia pasante de $50 \Omega$ nominal fue conectada al multímetro digital PROVA 803 para obtener la corriente que produce el fotodiodo. 


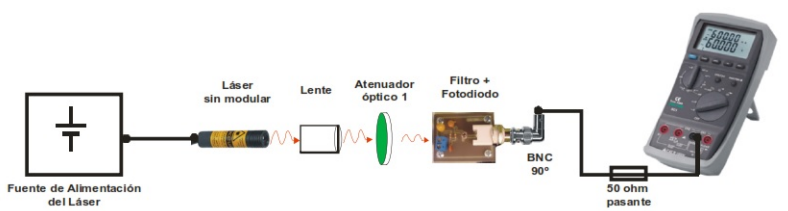

Fig. 4. Esquema del sistema de medición para caracterización de $I$ versus voltaje de polarización. El filtro óptico se cambia para lograr diferentes $S_{\text {med }}$.

Las curvas de $I$ vs $V$ para distintas $S_{\text {med }}$ se muestran en la Figura 5. Cabe recordar que el fabricante ha especificado al fotodiodo para una corriente de saturación inversa de hasta $100 \mu \mathrm{A}$ y en el presente trabajo se lo ha utilizado con corrientes máximas de alrededor de 1,20mA. Estos resultados resultan útiles para conocer los valores de corriente presentes a la salida del fotodiodo, como así también para asegurar que el modelo de continua del fotodiodo es válido aún fuera del área de especificación del fabricante.

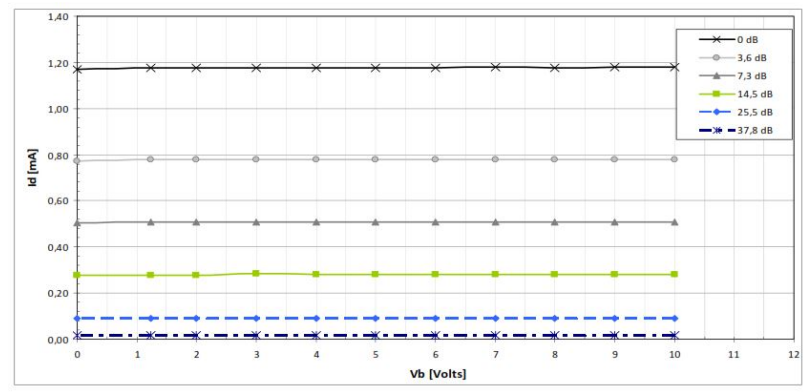

Fig. 5. Curvas de corriente de salida en función de la tensión de polarización inversa para diferentes intensidades medias de iluminación óptica

La respuesta dinámica de los fotodiodos en función de la intensidad luminosa media fue estudiada empleando el arreglo de la Figura 6. La profundidad de modulación fue de 1/50; cambiando la frecuencia de modulación y el valor de $S$. Para adaptar las impedancias se emplearon dos cargas pasantes externas de $50 \Omega$ de amplio ancho de banda. Todas las mediciones fueron realizadas sin ningún tipo de amplificación externa al fotodiodo.

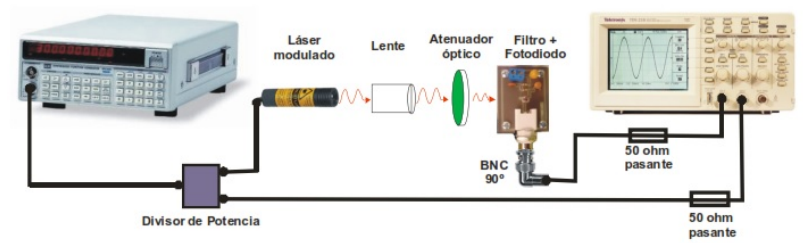

Fig. 6. Esquema del sistema de medición para determinar la respuesta dinámica

Los resultados obtenidos se presentan en las Figuras 7 y 8 , donde se observa la función transferencia del dispositivo, definida como la relación entre las tensiones de pico de la señal del generador y del circuito de fotodetección, en módulo y en fase respectivamente. En ambas figuras se puede apreciar una fuerte dependencia del módulo y la fase con la frecuencia al aumentar $S$. Es de notar que la frecuencia en la cual aparece alinealidad en la fase es aproximadamente $1 / 10 f_{3 d B}$, donde $f_{3 d B}$ es la frecuencia de corte del módulo.

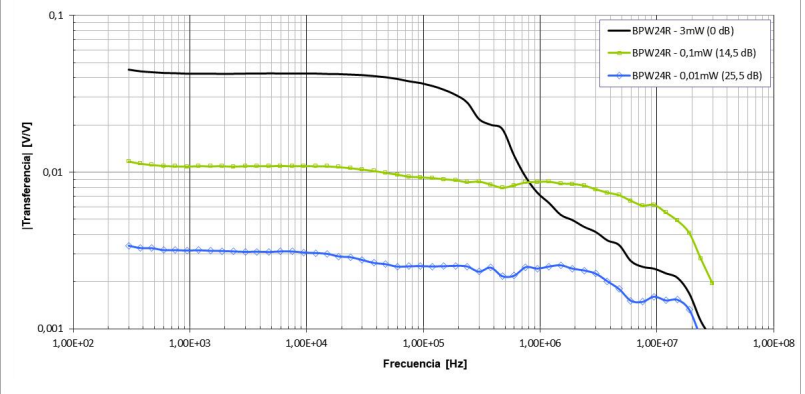

Fig. 7. Respuesta en frecuencia del BPW24R en módulo

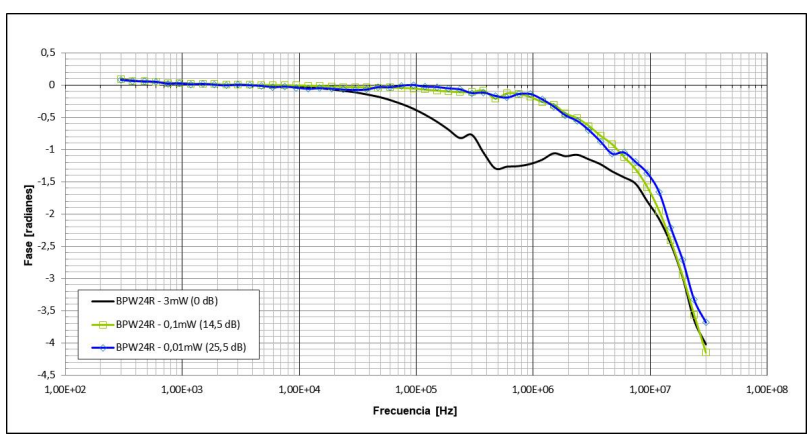

Fig. 8. Respuesta en frecuencia del BPW24R en fase

\section{Análisis}

Como se muestra en la Figura 7, para $S$ elevadas se manifiesta una distorsión en la fase a frecuencias de órdenes de magnitud menor que la de corte, provocando una deformación de la señal. Como se mencionó anteriormente, este resultado se debe a que en alta iluminación los efectos de la carga espacial perturban el campo eléctrico en la juntura, induciendo una respuesta no lineal.

En la Figura 8 se grafica la dependencia de la corriente de diodo (o saturación) en función de la frecuencia de corte (en -3dB) para el fotodiodo estudiado. A modo de referencia, se agregaron líneas de tendencia que indican la proporcionalidad a las cuales se aproximan y, con el fin de comparar el comportamiento observado con otras tecnologías, se incluyeron dos curvas adicionales que corresponden a fotodiodos PIN de superficie iluminada (SIPD) y fotodiodos de onda viajera (TWPD). Es importante mencionar que estas curvas adicionales se trazaron con datos extrapolados de estudios realizados sobre fotodetectores de alta frecuencia y alta intensidad [10]. Con el objetivo de poder comparar los resultados en una única escala, los datos de corriente y frecuencia fueron normalizados a la máxima frecuencia y corriente de cada caso. En el gráfico se observa que el fotodiodo BPW24R posee dos regiones diferenciadas según la dependencia en su frecuencia de corte, las cuales responden en forma aproximada a las proporcionalidades anteriormente mencionadas. Sin embargo, es importante notar que el fotodiodo cambia dicha dependencia en función de $S_{m e d}$. 


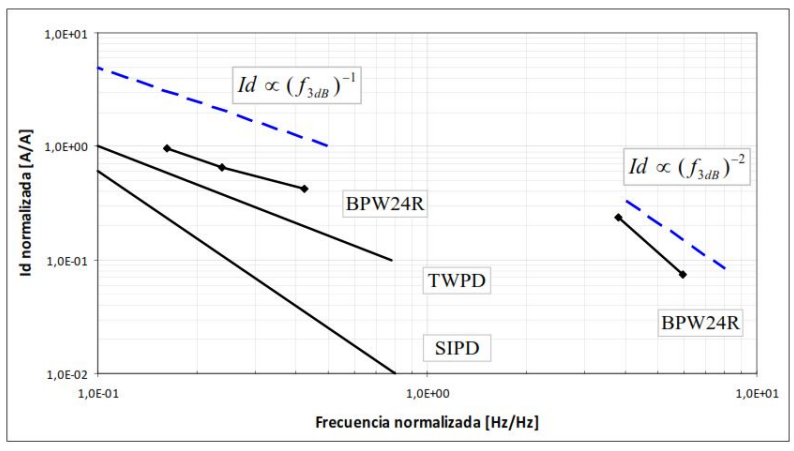

Fig. 9. Dependencia de la corriente de diodo respecto de la frecuencia de corte $(-3 d B)$ para el BPW24R, para diferentes intensidades medias

Todo dispositivo detector óptico se compone de dos partes: el transductor óptico con la electrónica de polarización, y la etapa de amplificación con la adaptación de impedancias adecuada. Un diseño es correcto cuando las limitaciones del dispositivo detector vienen dadas únicamente por los aspectos constructivos y procesos físicos inherentes al fotodetector.

Estos efectos deben ser tenidos en cuenta si se quiere reconstruir una señal luminosa arbitraria. Una opción posible para recuperar la señal es utilizar un filtro digital cuya respuesta impulsiva sea similar a la obtenida del sistema pero invertida en tiempo. Esta inversión en tiempo resulta en una respuesta en frecuencia equivalente, pero posee la ventaja que revierte la respuesta en fase, cancelando por lo tanto la distorsión mencionada.

\section{CONCLUSIÓN}

La mayoría de los dispositivos de detección de señales ópticas comerciales están caracterizados en condiciones de iluminación de baja intensidad, por lo que se hace difícil extrapolar su comportamiento en aplicaciones de alta intensidad sin un proceso experimental.

En este trabajo se muestra el comportamiento del fotodiodo PIN (BPW24R) al emplearlo en diferentes intensidades de luz. Se muestra que, si no se diseña un filtro digital específico, existe una limitación para la recuperación de señales arbitrarias, ya que al superar un cierto valor de intensidad media, se presenta una distorsión en módulo (por encima de la $f_{3 d B}$ ) y en fase (por encima de $1 / 10 f_{3 d B}$ ).

Al trabajar con altas intensidades de luz, cualquiera sea el experimento o estudio que se desee realizar, será sumamente importante hacer un análisis experimental del dispositivo detector a utilizar, sea este un producto comercial o un desarrollo ad-hoc armado en laboratorio para tal fin, con el objetivo de evidenciar su comportamiento y poder decidir si éste será útil en las condiciones experimentales de la aplicación.

\section{REFERENCIAS}

[1] R. H. Kingston, Detection of Optical and Infrared Radiation, 1st ed., ser. Springer Series in Optical Sciences. Springer-Verlag Berlin Heidelberg, 1978, vol. 10.

[2] R. J. Keyes, Ed., Optical and Infrared Detectors, ser. Topics in Applied Physics. Springer-Verlag, Berlin; Heidelberg New York, 1977, vol. 19.

[3] C.-K. Sun, I.-H. Tan, and J. E. Bowers, "Ultrafast transport dynamics of $\mathrm{p}$-i-n photodetectors under high-power illumination," IEEE Photonics Technology Letters, vol. 10, no. 1, pp. 135-137, Jan 1998.
[4] Y. Kuhara, Y. Fujimura, N. Nishiyama, Y. Michituji, H. Terauchi, and N. Yamabayashi, "Characterization and theoretical analysis of second-order intermodulation distortion of ingaas/inp p-i-n photodiode modules for fiber-optic catv." Journal of Lightwave Technology, vol. 15, no. 4, pp. 636-641, April 1997.

[5] W. Holzapfel, S. Neuschaefer-Rube, and M. Kobusch, "High-resolution, very broadband force measurements by solid-state laser transducers," Measurement, vol. 28, no. 4, pp. $277-291,2000 . \quad$ [Online]. Available: http://www.sciencedirect.com/science/article/pii/S0263224100000427

[6] M. Alessi, E. Zothner, E. Acosta, and M. Bisceglia, "Simultaneous measurement of electric birefringence, light scattering, and electric current for liquid systems," Review of Scientific Instruments, vol. 77, no. 12, p. 123103, 2006. [Online]. Available: http://dx.doi.org/10.1063/1.2403838

[7] S. Donati, Photodetectors: Devices, Circuits and Applications. Prentice Hall, Upper Saddle River, NY, 1999.

[8] J. E. Viallet, S. Mottet, L. L. Fjerou, and C. Boisrobert, "Photodiode for coherent detection: Modeling and experimental results," J. Phys. Colloques, vol. 49, pp. C4-321-C4-324, 1988. [Online]. Available: https://doi.org/10.1051/jphyscol:1988467

[9] S. Iezekiel, Microwave Photonics: Devices and Applications, ser. Wiley - IEEE. Wiley, 2009. [Online]. Available: https://books.google.com.ar/books?id=3NIy4Qv6PCcC

[10] M. C. Wu, T. Itoh, T. Chau, S. Islam, S. Mathai, A. Rollinger, and A. Nespola, "Recent progresses in high frequency, high power photodetectors," in International Topical Meeting on Microwave Photonics. Technical Digest (including High Speed Photonics Components Workshop) (Cat. No.98EX181), Oct 1998, pp. 237-239. 